\title{
Tracing Metallicity in High Redshift Quasars
}

\author{
Leah E. Simon and Fred Hamann
}

Department of Astronomy, University of Florida

211 Bryant Space Science Center, P.O. Box 112055

Gainesville, FL 32611, USA

\begin{abstract}
We present two ongoing studies of gas phase abundances around high redshift quasars. First, we examine broad emission line (BEL) metallicities for 29 quasars with 2.3 $<\mathrm{z}<4.6$ and far-infrared (far-IR) luminosities $\left(\mathrm{L}_{F I R}\right)$ from $10^{13.4}$ to $\leqslant 10^{12.2} \mathrm{~L}_{\odot}$, corresponding to star formation rates (SFRs) of 6740 to $\leqslant 1360 \mathrm{M}_{\odot} \mathrm{yr}^{-1}$. Quasar samples sorted by $\mathrm{L}_{F I R}$ might represent an evolutionary sequence if SFRs in quasar hosts generally diminish across quasar lifetimes. We create three composite spectra from rest-frame ultra-violet Sloan Digital Sky Survey spectra with increasing far-IR luminosity. We measure the N V $(\lambda 1240) / C$ IV $(\lambda 1550)$ and Si IV $(\lambda 1397)+\mathrm{O} \operatorname{IV}](\lambda 1402) / C \operatorname{IV}(\lambda 1550)$ emission line flux ratios for each composite and find uniformly high $(\sim 5-10$ times solar $)$ metallicities for the three composites, and no evidence for changes in metal enrichment with changes in ongoing SFR. Second, we present preliminary results from the largest ever survey of high resolution associated absorption line (AAL) region metallicities and physical properties in a sample of high redshift $(\mathrm{z}>3)$ quasars. This includes five quasars with previously known AALs at $\mathrm{z}>4$ and two well measured $\mathrm{z} \sim 3$ quasars with unusually rich absorption spectra. We determine well-constrained metallicities of about twice solar for five AAL systems. We find a range of lower limits for AAL metallicities in the $\mathrm{z}>4$ quasars from $1 / 100$ ths solar to 3 times solar. Overall, these results for typically super-solar gas-phase metallicities near quasars are consistent with evolutionary schemes where the major episodes of star formation in the host galaxies occur before the visibly luminous quasar phase. High SFRs (comparable to ULIRGs) in the host galaxies are not clearly linked to younger or chemically less mature quasar environments.
\end{abstract}

Keywords. techniques: spectroscopic, (galaxies:) quasars: absorption lines, (galaxies:) quasars: emission lines, galaxies: evolution

High redshift quasars are thought to represent an early stage of galaxy evolution, in which models by e.g., Dimatteo et al. (2008) and Hopkins et al. (2008) predict that major mergers trigger violent star formation and the rapid growth of a central super massive black hole. However, the timing of the quasar phase during a galaxy's evolution is less well understood: quasar feedback could quench, trigger, or have little effect on star formation in host galaxies. The gas-phase metal abundance works as a fossil record for the star formation in the host galaxy before the visible quasar epoch. Abundances have been probed in the near-quasar environment using broad emission lines (BELs) out to redshifts z $>6$ by e.g., Juarez et al. (2009), Nagao et al. (2006) and Dietrich et al. (2003), who consistently find metallicities near or above the solar value. This result suggests that there is always significant star formation before the quasar becomes visible.

We compare the metallicities in high-redshift quasars to the ongoing star formation rates (SFRs) in their host galaxies using measurements of BEL line flux ratios and far-infrared (far-IR) luminosities to constrain the late stages of galaxy-quasar evolution and the possible effects of quasar feedback on star formation. We measure BEL flux ratios, N V $(\lambda 1240) / \mathrm{C} \operatorname{IV}(\lambda 1550)$ and $\operatorname{Si} \operatorname{IV}(\lambda 1397)+\mathrm{O} \operatorname{IV}](\lambda 1402) / \mathrm{C} \operatorname{IV}(\lambda 1550)$, for three composite rest-frame ultraviolet spectra consisting of a sample of $292.3<\mathrm{z}<4.6$ Sloan Digital Sky Survey quasars grouped by far-IR luminosity, with $<L_{60}>=10^{46.90}, 10^{46.52}$ 
and $\leqslant 10^{46.21} \mathrm{erg} \mathrm{s}^{-1}$ for the three composites, corresponding to SFRs of 6740,2810 and $\leqslant 1360 \mathrm{M}_{\odot} \mathrm{yr}^{-1}$ if the far-IR is powered by star formation. We convert the flux ratios into metallicities using the theoretical relationship in which secondary enrichment processes increase the $\mathrm{N} / \mathrm{C}$ ratio as metallicity increases, and determine the average metallicity for each composite (e.g. Shields (1976)).

We find uniformly high $(\sim 5-10$ times solar $)$ metallicities for the three composite spectra. No significant variations in metal enrichment exist among the three $\mathrm{L}_{F I R}$ groups. By the time a quasar becomes visible, most of the gas enriching star formation is complete and any ongoing star formation does not contribute significantly to the enrichment (See Simon \& Hamann (2009)).

We investigate the galaxy-black hole formation relationship from a different perspective using the largest ever survey (others include Savaglio et al. (1994), Wampler et al. (1996)) of narrow associated absorption line (AALs, $\mathrm{v}_{\text {width }}<500 \mathrm{~km} \mathrm{~s}^{-1}$, forming within $12,000 \mathrm{~km} \mathrm{~s}^{-1}$ of the quasar emission velocity) metallicities and physical properties in a sample of high-redshift $(\mathrm{z}>3)$ quasars with Keck high resolution spectra in a range of near-quasar environments, including quasar outflows and host galaxy halos. We measure C IV, and H I optical depths, covering fractions, widths, column densities and obtain good ionization constraints, and determine well-constrained metallicities for five AAL systems in one $\mathrm{z}>3$ and two $\mathrm{z}>4$ quasars, and determine lower limits for metallicities for AALs in four other $\mathrm{z} \sim 4$ quasars.

Both BELs and AALs are composed of metal rich gas at all redshifts although metallicities derived from emission lines tend to be higher than those from absorption lines. The AALs have a broader range in metallicity, from only 100ths solar to up to 10 times solar. One $\mathrm{z} \sim 3.5$ quasar has AALs that are supersolar in 7 out of 9 systems (See Simon \& Hamann (2010)). Smaller velocity shifts and broader lines are more likely to be associated directly with the quasar as outflows, but we see no strong trend in metallicity with velocity shift or line width. The AALs with covering fraction less than one are more likely to be metal rich.

These super-solar metallicity results are consistent with previous observations and with evolutionary models, in which the quasar host galaxies experience significant star formation before the appearance of the visible quasar (Dimatteo et al. (2008), Hopkins et al. (2008)). High SFRs (comparable to ULIRGs) in the host galaxies are not clearly linked to younger or chemically less mature quasar environments.

\section{References}

Dietrich M., Hamann, F., Shields, J. C., Constantin, A., Heidt, J., Jäger, K., Vestergaard, M., \& Wagner, S. J. 2003, ApJ, 589, 722

DiMatteo, T., Colberg, J., Springel, V., Hernquist, L., \& Sijacki, D. 2008, ApJ, 676, 33

Hamann, F., Korista, K. T., Ferland, G. J., Warner, C., \& Baldwin, J. 2002, ApJ, 564, 592

Hopkins, P. F., Hernquist, L., Cox, T. J., \& Kereš, D. 2008, ApJS, 175, 356

Juarez, Y., Maiolino, R., Mujica, R., Pedani, M., Marinoni, S., Nagao, T., Marconi, A., \& Oliva, E. 2009, $A \mathscr{E} A, 494, \mathrm{~L} 25$

Nagao, T., Marconi, A., \& Maiolino, R. 2006, A\&A, 447, 157

Savaglio, S., D’Odorico, S., \& Møller, P. 1994, A\&A, 281, 331

Shields, G. A. 1976, ApJ, 204, 330

Simon, L. E., \& Hamann, F. 2009, in prep.

Simon, L. E., \& Hamann, F. 2010, in prep.

Wampler, E. J., Williger, G. M., Baldwin, J. A., Carswell, R. F., Hazard, C., \& McMahon, R. G. 1996, AESA, 316, 33 